\title{
Ce qu'il vous faut savoir pour votre formation postgraduée
}

\section{Christoph Hänggeli}

Avocat, administrateur responsable du Secrétariat de la formation prégraduée, postgraduée et continue (FPPC)

\section{Qui répond à mes questions en matière de formation postgraduée?}

Le Secrétariat de la formation prégraduée, postgraduée et continue (FPPC) de la FMH est l'instance compétente pour toute question relevant de la formation postgraduée et continue. Vous trouverez l'ensemble des informations les plus importantes sur notre site Internet (www. fmh.ch/awf). Nous répondons personnellement à toutes vos questions pendant les heures de bureau. Vous pouvez nous atteindre à l'adresse de correspondance.

L'aide-mémoire que vous pouvez télécharger sur notre site vous donne la vue d'ensemble sur la réglementation existante et les compétences dans le domaine de la formation prégraduée, postgraduée et continue [1].

\section{Quelles exigences dois-je satisfaire pour} obtenir un titre de formation postgraduée? Les principes généraux de la formation postgraduée des médecins sont précisés dans la Règlementation pour la formation postgraduée (RFP). En outre, il existe un programme pour chaque titre de médecin spécialiste et pour celui de «médecin praticien».

Prenez attentivement connaissance du programme de formation postgraduée que vous avez choisi, eu égard notamment aux exigences spécifiques de votre curriculum (catégories, séquences et répartition des années de formation, cours à suivre, publications à remettre).

La liste des établissements de formation reconnus indique les institutions auprès desquelles vous pouvez accomplir la formation postgraduée et les durées de formation maximales pouvant être validées. Important: toute activité effectuée dans des hôpitaux, des cliniques ou des cabinets médicaux qui ne figurent pas sur la liste n'est pas reconnue! Au moment de votre engagement, assurez-vous que l'on vous remet un contrat de travail écrit. Vérifiez au préalable qu'il s'agit bien d'un établissement de formation auprès duquel vous pouvez obtenir un certificat officiel délivré par la directrice ou le directeur.

\section{Puis-je aussi effectuer ma formation postgraduée à temps partiel?}

Oui, mais à certaines conditions: ne peuvent être validés que des engagements équivalant à un taux d'occupation de 50\% au moins. En outre, si aucune disposition spécifique ne figure dans le programme de formation postgraduée, le principe suivant s'applique: la moitié au plus de la formation postgraduée spécifique peut être accomplie à temps partiel. En revanche, les formations postgraduées non spécifiques peuvent être accomplies entièrement à temps partiel. De nombreux programmes de formation postgraduée admettent que la totalité de la formation soit accomplie à temps partiel.

\section{Puis-je suivre une formation postgraduée à l'étranger?}

Avant d'accepter un poste à l'étranger, sollicitez au préalable l'accord de la Commission des titres pour être certain que la validation de votre formation pourra se faire sans problème. En règle générale, toute formation suivie auprès d'un établissement de formation à l'étranger équivalent est reconnue comme partie de la formation continue réglementaire. Le candidat doit néanmoins suivre pendant au moins deux ans la formation postgraduée spécifique au titre dispensée par un établissement de formation reconnu en Suisse. Tenez compte des prescriptions particulières du programme de formation relatives à la définition de la formation postgraduée spécifique, à la classification des établissements de formation et à la validation des opérations. Pour certains titres de spécialiste mais aussi pour toutes les formations approfondies, la formation postgraduée peut être entièrement suivie à l'étranger [2].

Pour toute demande de titre, merci d'utiliser le formulaire électronique officiel sur notre site internet www.fmh.ch/awf.

\section{Y a-t-il une durée minimale pour la validation d'une période} de formation postgraduée?

Oui. Seules sont reconnues les périodes de formation postgraduée de six mois au moins accomplies auprès d'un même établissement de formation (en cas de formation à temps partiel: la durée est prolongée au prorata). Au maximum trois périodes courtes d'au moins trois mois sont admises par spécialité (pour chaque formation approfondie, une période courte peut être validée). L'assistanat en cabinet et le service militaire 
peuvent être validés à partir d'une période ininterrompue d'un mois déjà et ne comptent pas comme période courte.

\section{Puis-je faire valider une grossesse/} maternité comme formation postgraduée? Si votre certificat FMH fait mention d'absences en raison d'une grossesse/maternité, un rattrapage n'est pas nécessaire dans la mesure où le cumul des absences par discipline et par année n'excède pas huit semaines. A condition que vous ne dépassiez pas la limite maximale des absences selon les certificats FMH, la grossesse/ maternité peut, sur demande, également être validée indépendamment d'une période de formation précise. La réglementation détaillée se trouve à l'art. 31 de la Réglementation pour la formation postgraduée (RFP) [3].

\section{Mon assistanat au cabinet médical ou mon activité de remplaçant seront-ils validés?}

L'assistanat en cabinet et le remplacement seront validés dans le cadre défini par le programme de formation postgraduée. En outre, l'art. 34 de la Réglementation pour la formation continue est applicable.

\section{Puis-je faire valider une période de formation postgraduée simultanément pour deux titres de spécialiste différents?} Oui, c'est parfaitement possible. Deux années de médecine interne peuvent par exemple être simultanément validées pour les titres de spécialiste en médecine interne, médecine générale et cardiologie. L'acquisition de plusieurs titres de spécialiste s'en trouve facilitée.

\section{Dois-je réussir l'examen de spécialiste?} En principe oui! Dans certaines spécialités (surtout les formations approfondies) l'examen de spécialiste ou l'examen de formation approfondie vient d'être introduit. La question de savoir si vous ne devez que participer à l'examen ou si la réussite de celui-ci est exigée dépend de la spécialité choisie. Nous vous renvoyons à l'article détaillé paru sur la question dans le Bulletin des médecins suisses et figurant sur le site Internet du secrétariat FPPC [4]. Les délais pour l'examen de spécialiste et les modalités d'inscription sont publiés six mois avant la date de l'examen dans la rubrique Formation postgraduée $\rightarrow$ Examens de spécialiste sur le site internet. Un rappel paraît également dans le Bulletin des médecins suisses.

\section{Comment dois-je présenter} ma demande de titre?

La demande d'obtention d'un titre de spécialiste FMH doit être exclusivement présentée sur le site Internet de la FMH (www.fmh.ch/awf) au moyen du formulaire électronique de demande à l'attention de la Commission des titres. Au moyen de la liste de contrôle, assurez-vous d'avoir réuni l'ensemble des documents requis par le programme de formation postgraduée. Veillez à ne remettre que des photocopies bien lisibles de tous les certificats FMH requis, y compris des formulaires d'évaluation spécifique éventuels ainsi que des procès-verbaux d'évaluation s'y rapportant. Le secrétariat de la FPPC se verra malheureusement contraint de retourner tout dossier incomplet et tout document original! Les certificats FMH ne peuvent être validés que s'ils sont munis de votre signature ainsi que de celle du/ de la responsable autorisé(e) de l'établissement de formation reconnu. Si vous êtes dans l'impossibilité de remplir vous-même le formulaire de demande officiel sur notre site internet, c'est avec plaisir que nous vous aiderons. Le traitement de votre demande nécessitera toutefois plus de temps.

\section{Dois-je envoyer chaque année mes certificats de formation à la FMH?}

Non. N'envoyez vos certificats FMH qu'après avoir rassemblé l'ensemble des justificatifs requis par le programme de formation. Toute période de formation postgraduée doit être sanctionnée par un certificat FMH reconnu et par les formulaires d'évaluation spécifiques éventuels et le cas échéant par un journal de bord («logbook»). Le responsable de la formation postgraduée procède au moins une fois par année à un entretien d'évaluation, dont les résultats sont consignés dans les protocoles officiels et/ou dans le journal de bord.

\section{Quand un programme de formation postgraduée est-il révisé?}

La médecine est en constante évolution et les exigences spécifiques aux différents programmes de formation postgraduée aussi. Vérifiez régulièrement si le curriculum que vous avez choisi a été actualisé ou non (www.fmh.ch/awf). En règle générale, les nouvelles exigences sont introduites moyennant une période transitoire de trois à cinq années. En d'autres termes, le candidat qui achève sa formation postgraduée dans un délai de trois ans à partir de la mise en vigueur du nou- 
veau programme peut le faire selon les dispositions de l'ancien programme. Passé ce délai, s'appliquent les exigences du nouveau programme.

\section{Dois-je être membre de la FMH} pour acquérir un titre de spécialiste, une formation approfondie ou une attestation de formation complémentaire?

L'affiliation à la FMH n'est pas nécessaire pour acquérir les différents titres postgrades fédéraux. Il en va autrement pour les formations approfondies, les attestations de formation complémentaire et les titres en chirurgie de la main et en neuropathologie, qui ne sont pas régis par la loi sur les professions médicales (LPMéd) et qui sont, à quelques exceptions près, décernés en exclusivité aux membres FMH. De même, l'affiliation à une société de discipline médicale est facultative. Nous vous invitons cependant à vous renseigner sur les avantages liés à une adhésion.

\section{En tant que membre de la FMH,} dois-je aussi verser des émoluments?

Veuillez vous référer au tarif des émoluments sur notre site Internet [5]. En tant que membre de la FMH, vous profitez des prestations gratuites suivantes:

- renseignements et conseils pour toutes les questions de formation postgraduée (par exemple validation de la formation accomplie à l'étranger);

- élaboration d'un plan de formation postgraduée, sur la base duquel la Commission des titres évalue la formation déjà accomplie et indique les exigences qui doivent encore être satisfaites.

15. Puis-je aussi évaluer mon établissement de formation postgraduée?

La FMH procède chaque année à une enquête auprès de l'ensemble des médecins-assistants afin d'évaluer dans le détail les établissements de formation. Participez activement à cette enquête afin que la FMH puisse améliorer en permanence sa formation postgraduée!

\section{Références}

1 Aide-mémoire: www.fmh.ch/awf $\rightarrow$ Formation postgraduée $\rightarrow$ Europe / Accords bilatéraux.

2 Article «Weiterbildung im Ausland leicht gemacht»: www.fmh.ch/awf $\rightarrow$ Formation postgraduée $\rightarrow$ Bases légales $\rightarrow$ Publications.

3 Interprétation de l'art. 31 RFP: www.fmh.ch/awf $\rightarrow$ Formation postgraduée $\rightarrow$ Bases légales.

4 Article «Quels critères pour l'examen de spécialiste?»: www.fmh.ch/awf $\rightarrow$ Formation postgraduée $\rightarrow$ Examen de spécialiste.

5 Tarif des émoluments: www.fmh.ch/awf $\rightarrow$ Formation postgraduée $\rightarrow$ Bases légales $\rightarrow$ Publications. 